\title{
An Android Application for Leaf-based Plant Identification
}

\author{
Sofiene Mouine ${ }^{\dagger}$, Itheri Yahiaoui ${ }^{\ddagger \ddagger}$, Anne Verroust-Blondet ${ }^{\dagger}$, Laurent Joyeux ${ }^{\dagger}$, \\ Souheil Selmi ${ }^{\dagger}$, Hervé Goëau ${ }^{\dagger}$ \\ ${ }^{\dagger}$ Inria Paris-Rocquencourt 78153 Le Chesnay, France \\ ‡ CReSTIC Reims University, 51687 Reims, France

\begin{abstract}
sofiene.mouine@inria.fr, itheri.yahiaoui@univ-reims.fr, anne.verroust@inria.fr, laurent.joyeux@inria.fr, souheil.selmi@inria.fr, herve.goeau@inria.fr
\end{abstract}

\begin{abstract}
This paper presents an Android application for plant identification. The system relies on the observation of leaf images. Unlike other mobile plant identification applications, the user may choose the leaf characters that will guide the identification process. For this purpose, two kinds of descriptors are proposed to the user: a shape descriptor based on a multiscale triangular representation of the leaf margin and a descriptor of the salient points of the leaf. The application achieves good identification accuracy and provides Android users a useful system for plant identification.
\end{abstract}

\section{Categories and Subject Descriptors}

H.5.1 [Information Interfaces and Presentations]: Multimedia Information Systems

\section{General Terms}

Algorithms, Experimentations

\section{Keywords}

Android application, plant identification, leaf descriptor, shape representation, local descriptor

\section{INTRODUCTION AND MOTIVATION}

Identifying plants is a challenging task considering the large number of existing species in the world. The interspecies similarity and the intra-species variability make the identification task particularly difficult and time consuming. We present here a practical plant identification tool based on the visual information provided by leaves. Our tool is an Android application which has been developed within the $\mathrm{Pl@ntNet^{1 }}$ project. The application is intended for mobile devices to allow a user to identify plants on the spot. It can also be used as an observation collector tool to enrich the knowledge database. Unlike similar applications where the identification is a black-box processing, our mobile system enables the user to choose a specific leaf descriptor (margin, venation points, both of them) that will be used as the basis of identification. Within this application, we focus on a

Copyright is held by the author/owner(s). ICMR'13, April 16-20, 2013, Dallas, Texas, USA. ACM 978-1-4503-2033-7/13/04.

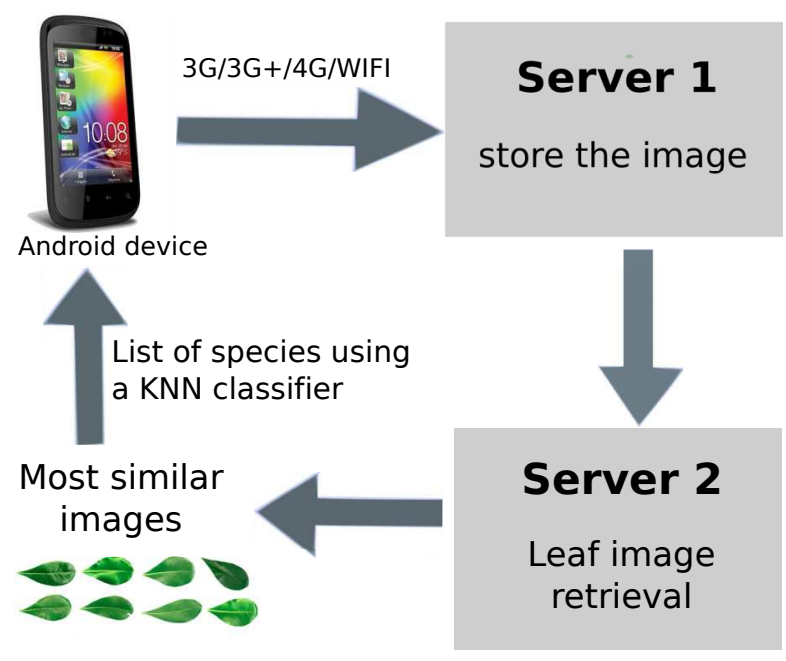

Figure 1: Plant identification process

set of leaf descriptors that have shown a noteworthy performance on several public leaf databases. In addition to leaf species identification, the user will also be able to compare the effectiveness of the descriptors for a given leaf image.

\section{PLANT IDENTIFICATION PROCESS}

The plant identification process is summarized in Figure 1. The user captures a leaf image with an Android device. Hypotheses made on the taken images are the same as those in [4]: the image contains a centred single leaf on a uniform background. Before launching the identification, the user has to select a leaf character that will be the basis of the identification (margin, venation points). Then, the leaf image is sent to a primary intermediate server with a degraded quality to save bandwidth. However, this step requires an adequate bandwidth. For this purpose, a 3G network or later networks are required. The role of the primary server is to store the image in order to broaden the knowledge about plant species. The identification step is performed on the second server using the descriptor previously selected by the user. The descriptors have been embedded in the IKONA content-based image retrieval system [1]. Finally, a

\footnotetext{
${ }^{1}$ http://www.plantnet-project.org/
} 
ranked list of leaf species is returned and displayed on the Android device (cf. Figure 2). We use a knowledge database, which is off-line indexed using each of the descriptors suggested to the user. On the other hand, the signature of the image sent by the user is computed on-line and a large scale matching algorithm returns the most similar images [3]. A KNN Classifier is then used to build a list of species.

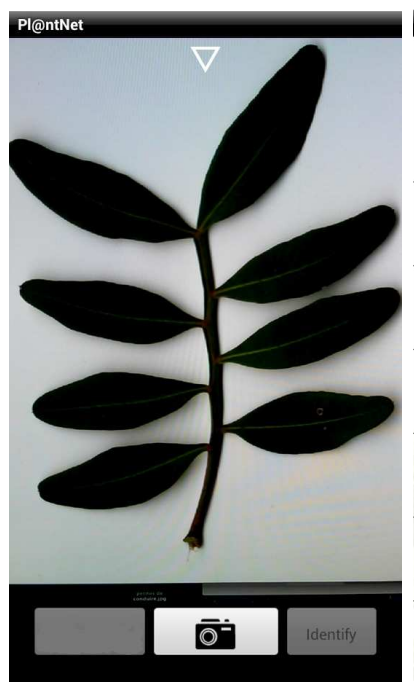

(a)

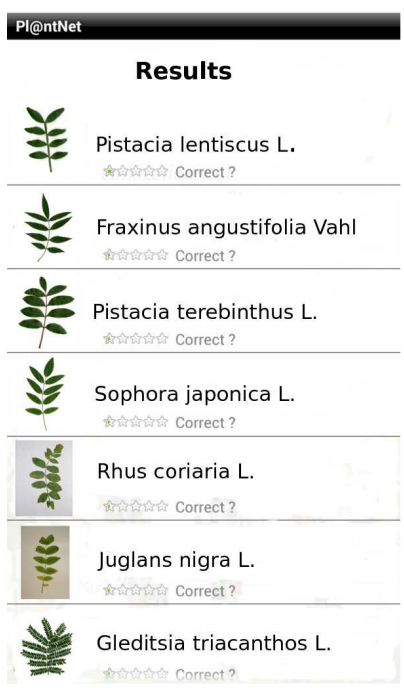

(b)
Figure 2: Screenshots of the Android application (a) Leaf image taken on a uniform background (b) Identification results. A ranked list of species is returned

\subsection{Knowledge database}

To identify the species of a leaf image, a retrieval technique is used. We need to have a knowledge database, i.e. a collection of annotated leaf images where a plant species is associated with each leaf image. This database will be used to find the most similar images to a query image. To do so, we use a subset of the training set of ImageCLEF 2012 plant identification task [2] freely available ${ }^{2}$ : the training sets of scans and scan-like images are merged into one to form our knowledge database. It contains 6698 images belonging to 122 species. Thus, our Android application make it possible to identify leaves from 122 different species.

\subsection{Architecture}

The linkage between the GUI on the mobile device and the identification system on the second server is done by a RESTful java web service which offers an attractive scalable computing architecture. In our case, our RESTful identification web service can be either a server or a client. It plays the role of a server when it receives the leaf image taken by the user. It is a client when it interfaces the identification server using the selected descriptor. The data exchange between the web service and the identification server is ensured via sockets. Besides the abstraction of the architecture, the web service allows simple communication between the Android device and our identification system.

\footnotetext{
${ }^{2}$ http://imedia-ftp.inria.fr:8080/imageclef2012/ImageCLE F2012PlantIdentification TaskFinalPackage.zip
}

\subsection{Plant identification methods}

Two kinds of methods are used within the Android application:

- A shape-based approach that describes the leaf margin using a multiscale triangular representation [6].

- A shape context based descriptor $S C 2$ that represents the salient points of the leaf (essentially venation points) in the context defined by the leaf boundary [5].

- The combination of the methods mentioned above by a late fusion algorithm.

These techniques have shown their effectiveness for leaf image retrieval. The $S C 2$ descriptor was tested on the ImageCLEF2011 leaf dataset. The shape-based approach using multiscale triangles has been evaluated on four public leaf datasets: Swedish, Flavia, ImageCLEF 2011 and 2012. It has also shown good robustness to partial occlusion. The identification results given by these descriptors can be found in $[5,6]$.

\section{CONCLUSION}

In this paper, an Android application for leaf species identification has been presented. It is based on a set of leaf descriptors that have given promising results on leaf datasets. The accuracy of the identification makes this application useful to amateur stakeholders as well as experts. Future work aims to expand the knowledge database by including leaf images from other species.

\section{Acknowledgements}

This research has been conducted with the support of the Agropolis Foundation through the Pl@ntNet project.

\section{REFERENCES}

[1] N. Boujemaa, J. Fauqueur, M. Ferecatu, F. Fleuret, V. Gouet, B. Lesaux, and H. Sahbi. Ikona: Interactive specific and generic image retrieval. In International workshop on Multimedia Content-Based Indexing and Retrieval (MMCBIR), 2001.

[2] H. Goëau, P. Bonnet, A. Joly, I. Yahiaoui, D. Barthélémy, N. Boujemaa, and J.-F. Molino. The IMAGECLEF 2012 Plant identification Task. In CLEF 2012, Rome, Italy, Sept. 2012.

[3] A. Joly and O. Buisson. Random maximum margin hashing. In $C V P R$, pages 873-880, 2011.

[4] N. Kumar, P. N. Belhumeur, A. Biswas, D. W. Jacobs, W. J. Kress, I. C. Lopez, and J. V. B. Soares. Leafsnap: A computer vision system for automatic plant species identification. In 12th European Conference on Computer Vision (ECCV 2012), pages 502-516, Florence, Italy, Oct. 2012.

[5] S. Mouine, I. Yahiaoui, and A. Verroust-Blondet. Advanced shape context for plant species identification using leaf image retrieval. In Proceedings of the 2nd ACM International Conference on Multimedia Retrieval, pages 49:1-49:8, 2012.

[6] S. Mouine, I. Yahiaoui, and A. Verroust-Blondet. A shape-based approach for leaf classification using a multiscale triangular representation. In Proceedings of the 3rd ACM International Conference on Multimedia Retrieval, 2013. 